\title{
Proposed use of spatial mortality assessments as part of the pesticide evaluation scheme for vector control
}

\author{
Beatriz Mosqueira ${ }^{*}$, Joseph Chabi ${ }^{2}$, Fabrice Chandre ${ }^{3}$, Martin Akogbeto ${ }^{2}$, Jean-Marc Hougard ${ }^{3}$, \\ Pierre Carnevale ${ }^{4}$ and Santiago Mas-Coma ${ }^{1}$
}

\begin{abstract}
Background: The WHO Pesticide Evaluation Scheme to evaluate the efficacy of insecticides does not include the testing of a lethal effect at a distance. A tool was developed to evaluate the spatial mortality of an insecticide product against adult mosquitoes at a distance under laboratory and field conditions. Operational implications are discussed.

Methods: Insecticide paint, Inesfly 5A IGR ${ }^{T M}$, containing two organophosphates (OPs): chlorpyrifos and diazinon, and one insect growth regulator (IGR): pyriproxyfen, was the product tested. Laboratory tests were performed using "distance boxes" with surfaces treated with one layer of control or insecticide paint at a dose of $1 \mathrm{~kg} / 6 \mathrm{sq} \mathrm{m}$. Field tests were conducted up to 12 months in six experimental huts randomly allocated to control or one or two layers of insecticide paint at $1 \mathrm{~kg} / 6 \mathrm{sq} \mathrm{m}$. All distance tests were performed using reference-susceptible strains of Anopheles gambiae and Culex quinquefasciatus left overnight at a distance of $1 \mathrm{~m}$ from control or treated surfaces.

Results: After an overnight exposition at distances of $1 \mathrm{~m}$, field and laboratory evaluations at 0 months after treatment (T0) yielded 100\% mortality rates on surfaces treated with one layer at $1 \mathrm{~kg} / 6 \mathrm{sq} \mathrm{m}$ against susceptible strains of An. gambiae and $C x$. quinquefasciatus. Testing for long-term efficacy in the field gave mortality rates of 96-100\% after an overnight exposition at a distance of $1 \mathrm{~m}$ for up to 12 months in huts where a larger volume was treated (walls and ceilings) with one or two layers of insecticide paint.

Conclusion: A comprehensive evaluation of the full profile of insecticide products, both upon contact and spatially, may help rationalize vector control efforts more efficiently. Treating a large enough volume may extend a product's mortality efficacy in the long-term, which contact tests would fail to assess. It is hereby proposed to explore the development of cost effective methods to assess spatial mortality and to include them as one additional measurement of insecticide efficacy against mosquitoes and other arthropod vectors in WHOPES Phase I and Phase II studies.
\end{abstract}

Keywords: Vector control, WHOPES, Insecticide-treated nets (ITNs), Long-lasting insecticidal nets (LLINs), Indoor residual spraying (IRS), Insecticide paint, Mass effect

\section{Background}

Vector-borne diseases, such as malaria and dengue, are among the major causes of morbidity and mortality and significantly impede the economic and social development of many countries, predominantly in tropical areas, although not only. In temperate regions, West Nile virus,

\footnotetext{
* Correspondence: bmosqueira@yahoo.com

${ }^{1}$ Departamento de Parasitologia, Facultad de Farmacia, Universidad de Valencia, Av Vicent Andrés Estellés s/n, Burjassot 46100, Valencia, Spain Full list of author information is available at the end of the article
}

dengue, leishmaniasis and chikungunya, among other vector-borne diseases, are also causing an increasing burden.

Control strategies rely mostly on vector control using insecticides, treatment using drugs, improving people's dwellings/modifying the environment, education, and the creation of new vaccines. A promising intervention strategy involves genetic control of the vectors [1]. The strategies chosen will depend on several factors, such as resistance to insecticides, availability of treatment and/or resistance to available drugs, difficulties in developing a vaccine, existence of operational genetic control programmes, and long-

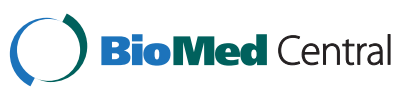


term sustainability. A combination of the above disease control strategies will increase chances to succeed.

Vector control is one of these strategies and remains a key player in the control of major endemic and epidemic vector-borne diseases such as malaria [2,3]. The official World Health Organization Pesticide Evaluation Scheme (WHOPES) guidelines for the evaluation of the efficacy of insecticides $[4,5]$ take into consideration products' impact on mortality, blood feeding, deterrence and repellence. Tests currently used include classical WHO contact bioassays $[6,7]$, tunnel tests $[8,9]$ and early morning collections in experimental huts $[10,11]$. These tests provide key information on the impact of insecticide products, such as long-lasting insecticidal nets (LLINs) or indoor residual spraying (IRS), upon contact both in the laboratory and the field, but does not provide information on the possible lethal effect at a distance.

Since even highly endophilic mosquitoes or other arthropod vectors are not always in contact with an insecticide-treated surface before biting a human or animal host, especially on pyrethroid-treated surfaces due to its irritant effect, it is desirable to evaluate the lethal effect spatially, that is, at a distance, without the mosquitoes ever entering into contact with an insecticide-treated surface.

Several studies on the community effect of ITNs on malaria indicate the presence of a beneficial mass effect [12-20]. A mass effect of IRS has also been documented in a number of trials [21].

In this study, distance tests were performed in the laboratory using "distance boxes", and in the field. In the field, evaluations were done in addition to WHO bioassays and early morning collections in experimental huts. The product evaluated consisted of an insecticide paint, Inesfly $5 \mathrm{~A} \mathrm{IGR}^{\mathrm{m}}$, composed of two organophosphates (OPs): chlorpyriphos (1.5\%) and diazinon (1.5\%), and an insect growth regulator (IGR): pyriproxyfen $(0.063 \%)$. The product was a white vinyl paint with an aqueous base. Active

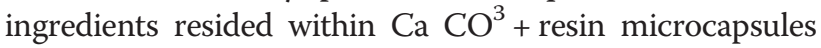
ranging from one to several hundred micrometres in size. The formulation allows a gradual release of active ingredients, increasing its durability. Toxicology studies performed so far support the product's safety [22-24]. Inesfly $5 \mathrm{~A} \mathrm{IGR}^{\text {mat }}$ had been evaluated previously under experimental conditions against the Chagas disease vector Triatoma infestans [25,26], Classical WHOPES tests were also performed on Inesfly $5 \mathrm{~A} \mathrm{IGR}^{\mathrm{m}}$ in the laboratory (Phase I) against 100\% OP-resistant Culex quinquefasciatus [27] and in the field (Phase II) against local wild pyrethroidresistant populations of the major malaria vector, Anopheles gambiae, and pest mosquito, Cx. quinquefasciatus [28]. In parallel to the standard Phase I evaluations [27], it was decided to explore the idea of a possible efficacy at a distance by exposing mosquitoes to metal-treated surfaces at distances of $3 \mathrm{~cm}, 40 \mathrm{~cm}$ and $100 \mathrm{~cm}$. Mortalities at shorter distance were almost the same as the ones upon contact (unpublished results). It was thus decided to test spatial mortality at distances of $100 \mathrm{~cm}$ from cementtreated surfaces so as to reproduce the same test on experimental huts during Phase II evaluations. The objective of this paper is to propose the use of spatial mortality tests as part of the WHOPES in the light of results obtained in the laboratory (Phase I) using "distance boxes" and in the field (Phase II) in experimental huts.

\section{Methods}

\section{Phase I - laboratory tests using distance boxes}

Two identical wooden boxes were built, one for control and one for treatment. The size of each wooden box was $50 \mathrm{~cm}$ wide $\times 50 \mathrm{~cm}$ high, length $100 \mathrm{~cm}$ with two horizontal slits of $4 \mathrm{~cm} \times 50 \mathrm{~cm}$ on each side. The two horizontal slits were placed in the middle of each side of the box to allow air to flow. Wood was chosen as a material readily available and easy to work with. One end was left open and is where mosquitoes were placed inside $150 \mathrm{ml}$ tubes. The other end was closed by a cement surface $50 \mathrm{~cm} \times 50 \mathrm{~cm}-$ cement was chosen to reproduce the material experimental huts were made of. The box used as control had a cement surface with no paint. The box used for treatment had a cement surface with of one layer of Inesfly $5 \mathrm{~A} \mathrm{IGR}{ }^{\mathrm{mi}}$ insecticide-paint at $1 \mathrm{~kg} / 6 \mathrm{sq} \mathrm{m}$. Boxes were placed in a closed room at $80 \pm 10 \%$ relative humidity and $27 \pm 2^{\circ} \mathrm{C}$ temperature.

Unfed females of An. gambiae Kisumu and Cx. quinquefasciatus S-Lab, three to five days old, reared at the Centre de Recherche Entomologique de Cotonou (CREC) insectarium, and susceptible to all insecticides, were used. Mosquitoes were introduced in four $150 \mathrm{ml}$ tubes with mosquito netting at both ends to protect them from scavengers but allow air through. Honey juice-soaked cotton was introduced in each tube to prevent females from starvation. Four replicates were made with 15 females each, giving a total of 60 females per surface per test. Tubes were placed horizontally at the edge of the box at $1 \mathrm{~m}$ from the cement surface from 19:00 to 07:00. The following morning, females were taken to the insectarium for delayed mortality assessments after 24 hours at $80 \pm 10 \%$ relative humidity and $27 \pm 2^{\circ} \mathrm{C}$ temperature. Distance testing was done only at 0 months after treatment (T0) under laboratory conditions.

\section{Phase II - field tests in experimental huts in Benin}

Inesfly $5 \mathrm{~A} \mathrm{IGR}{ }^{\mathrm{Tw}}$ was evaluated in six experimental huts at the Ladji station in Cotonou (south of Benin) [28]. Experimental huts were built following the West African-style hut model [29]. Huts were treated with one or two layers of insecticide paint at $1 \mathrm{~kg}$ commercial product $/ 6 \mathrm{sq} \mathrm{m}$, that is $0,51 \mathrm{~g}$ a.i. per sq $\mathrm{m}$. Based on huts' dimensions, $3.4 \mathrm{~kg}$ of paint were applied on walls per layer, and $1.0 \mathrm{~kg}$ 
on ceilings. Huts treated with two layers had the first layer diluted in $20 \%$ water following recommendations of the manufacturer. The overall random disposition of huts was: H1: Control 1 - no paint; H2: Control 2 - two layers of control paint on walls and ceiling; H3: one layer of insecticide paint on walls; H4: one layer of insecticide paint on walls and ceiling; H5: two layers of insecticide paint on walls; and H6: two layers of insecticide paint on walls and ceiling.

Unfed females of An. gambiae Kisumu and Cx. quinquefasciatus S-Lab, three to five days old, reared at the CREC insectarium, and susceptible to all insecticides, were used. A total of 60 females were introduced into four tubes of $150 \mathrm{ml}$, with 15 females per tube. Mosquito netting was placed at both ends to allow air through. Honeysoaked cotton was introduced to ensure that females did not die from starvation. Tubes containing females were placed inside the hut, on the floor, horizontally from 19:00 to $07: 00$, at a distance of $1 \mathrm{~m}$ from two perpendicular walls inside the hut and $1.90 \mathrm{~m}$ from the ceiling. The following morning, females were taken to the insectarium for delayed mortality assessment after 24 hours at $80 \pm 10 \%$ relative humidity and $27 \pm 2{ }^{\circ} \mathrm{C}$ temperature. Tests were performed again 12 months after treatment.

Results from laboratory and field distance tests were analysed using Epi-Info 6 . When values were $<5$, Fisher exact tests were used.

\section{Results}

Phase I - laboratory tests using distance boxes

Distance boxes yielded $100 \%$ mortality at 0 months after treatment against both $A n$. gambiae Kisumu and $C x$. quinquefasciatus S-Lab (Table 1). Compared to control, mortality rates were significantly different for the treated surface $\left(\mathrm{p}<10^{-6}\right)$.

\section{Phase II - field tests in experimental huts in Benin}

Under field conditions at T0, all huts, regardless of the surface treated and the number of layers, yielded 100\% mortality against both $A n$. gambiae Kisumu and $C x$. quinquefasciatus S-Lab (Table 1). Twelve months after treatment, mortality rates observed at the huts where a larger volume was treated with one or two layers of paint were $98.4 \%$ for $A n$. gambiae Kisumu and $96.2 \%$ for $C x$. quinquefasciatus S-Lab (Table 2). Mortality rates in the hut treated on only walls with one layer of insecticide paint was lower than in the other three huts: $36 \%$ mortality against susceptible An. gambiae Kisumu and 60\% against susceptible $C x$. quinquefasciatus S-Lab $\left(\mathrm{p}<10^{-6}\right)$, though still higher than control $\left(\mathrm{p}<10^{-6}\right)$.

\section{Discussion}

The results obtained in the laboratory and the field were similar at T0: $100 \%$ mortality rates were observed on surfaces treated with one layer of insecticide paint at the recommended dose of $1 \mathrm{~kg} / 6 \mathrm{sq}$ m against susceptible $A n$. gambiae Kisumu and Cx. quinquefasciatus S-Lab. Female mosquitoes were never in contact with the treated or control surfaces. A 1-m distance was respected in all cases for all repeats. The uniformity of results suggests distance boxes could be a useful and simple approach for testing the lethal efficacy of insecticide products at a distance in the laboratory during Phase I evaluations, but more data is needed. The distance of one metre was chosen because of the small size of experimental huts and of West African homes in general. The distance of one metre is thus proposed as an initial step, nevertheless the distance may be adapted depending on the nature of the insecticide (ie. vapour pressure) and the support used (ie. LLINs, IRS, DL, paint). The size of the dwellings to be treated may also play a role in deciding the distance to be tested - if large halls in schools, airports or hospitals are treated, it may be of interest to test for spatial mortality efficacy at greater distances.

To test for long-term spatial mortality efficacy, distance tests were performed again 12 months after treatment during Phase II studies in the field: spatial mortality after an overnight exposition at distances of $1 \mathrm{~m}$ remained high, 96-98\%, against susceptible An. gambiae Kisumu and $C x$. quinquefasciatus S-Lab in huts with one layer of insecticide paint if both, walls and ceiling were treated. On the other hand, huts treated with one layer but on walls only (not ceilings) performed less well, 36\% mortality against susceptible An. gambiae Kisumu and 60\% against susceptible $C x$. quinquefasciatus S-Lab after 12 months. That is, provided that a large enough volume was treated, huts with one layer performed as well as huts with two layers despite the difference in dose - this finding was referred to as the "volume effect". This notion of volume effect seemed to be supported by results obtained during Phase II early morning collections in the field: A volume effect

Table 1 Comparison of phase I and phase II spatial mortality rates in control and treated surfaces

\begin{tabular}{|c|c|c|c|}
\hline $\begin{array}{l}\text { Cement tested at a } \\
\text { distance of } 1 \mathrm{~m} \text { at T0 }\end{array}$ & Control & $\begin{array}{l}\text { One layer IP at } 1 \mathrm{~kg} / 6 \mathrm{sq} \mathrm{m} \\
\text { in distance box - phase I }\end{array}$ & $\begin{array}{l}\text { One layer IP at } 1 \mathrm{~kg} / 6 \mathrm{sq} \mathrm{m} \\
\text { in experimental huts - phase II }\end{array}$ \\
\hline An. gambiae Kisumu & $0^{\mathrm{a}}$ & $100^{\mathrm{b}}$ & $100^{b}$ \\
\hline Cx. quinquefasciatus S-Lab & $0^{a}$ & $100^{\mathrm{b}}$ & $100^{\mathrm{b}}$ \\
\hline
\end{tabular}

Delayed 24-hour mortality of Anopheles gambiae Kisumu and Culex quinquefasciatus S-Lab after overnight exposure at a distance of $1 \mathrm{~m}$ in distance boxes in laboratory, and in experimental huts in the field.

$I P$, Insecticide paint, TO, 0 months after treatment. Values in the same row sharing a letter superscript do not differ significantly (P $\geq 0.05)$. 
Table 2 Phase II spatial long-term mortality rates in control and treated experimental huts

\begin{tabular}{|c|c|c|c|c|c|c|c|}
\hline $\begin{array}{l}\text { Phase II - cement tested } \\
\text { at a distance of } 1 \mathrm{~m} \\
\text { at T0 and T12 }\end{array}$ & Timepoint & Control 1 & $\begin{array}{l}\text { Control } 2 \text { two layers } \\
\text { of control paint on } \\
\text { walls and ceiling }\end{array}$ & $\begin{array}{l}\text { One layer IP } \\
\text { on walls at } \\
1 \mathrm{~kg} / 6 \mathrm{sq} \mathrm{m}\end{array}$ & $\begin{array}{l}\text { One layer IP on } \\
\text { walls and ceiling } \\
\text { at } 1 \mathrm{~kg} / 6 \mathrm{sq} \mathrm{m}\end{array}$ & $\begin{array}{l}\text { Two layers IP } \\
\text { on walls at } \\
1 \mathrm{~kg} / 6 \mathrm{sq} \mathrm{m}\end{array}$ & $\begin{array}{l}\text { Two layers IP on } \\
\text { walls and ceiling } \\
\text { at } 1 \mathrm{~kg} / 6 \mathrm{sq} \mathrm{m} \\
\end{array}$ \\
\hline An. gambiae & T0 & $0^{a}$ & $3.4^{a}$ & $100^{b}$ & $100^{b}$ & $100^{b}$ & $100^{b}$ \\
\hline Kisumu & $\mathrm{T} 12$ & $1.5^{\mathrm{a}}$ & $3^{a}$ & $35.6^{\mathrm{b}}$ & $98.4^{c}$ & $100^{c}$ & $100^{c}$ \\
\hline \multirow[t]{2}{*}{ Cx. quinquefasciatus S-Lab } & TO & $8.3^{\mathrm{a}}$ & $0^{\mathrm{a}}$ & $100^{b}$ & $100^{b}$ & $100^{b}$ & $100^{b}$ \\
\hline & $\mathrm{T} 12$ & $1.8^{\mathrm{a}}$ & $3^{\mathrm{a}}$ & $60^{\mathrm{b}}$ & $96.2^{c}$ & $100^{c}$ & $100^{c}$ \\
\hline
\end{tabular}

Delayed 24-hour mortality of Anopheles gambiae Kisumu and Culex quinquefasciatus S-Lab after overnight exposure at a distance of $1 \mathrm{~m}$ from two perpendicular walls in experimental huts in the field.

$I P$, Insecticide paint, $T 0$ and $T 12,0$ and 12 months after treatment.

Values in the same row sharing a letter superscript do not differ significantly $(P \geq 0.05)$.

was observed during early morning collections performed in experimental huts in Ladji, south of Benin, with the same insecticide paint [28]. Likewise, a volume effect was observed when testing the efficacy of a pyrethroid-based insecticide paint against pyrethroid-susceptible populations of An. gambiae and Cx. quinquefasciatus during a Phase II study in experimental huts in the north of Benin: female mosquitoes exposed at a distance of $1 \mathrm{~m}$ from treated surfaces had a significantly higher mortality rate 12 months after treatment in huts where both walls and ceiling were treated even if with just one layer of paint, but not in huts with one layer on walls only [Mosqueira B, Chabi J, Soukou KB, Akogbeto M, Carnevale P, Corbel V, Mas-Coma S: Laboratory and field efficacy of a pyrethroidbased insecticide paint against insecticide-susceptible and resistant malaria-transmitting mosquitoes, in preparation]. Curiously, an irritant and deterrent effect was observed when comparing treated huts to control. Predictably, WHO contact bioassays failed to detect a volume effect: as far as WHO contact bioassays went, only the dose applied counted - two layers performing consistently better than one layer. Hence, Phase I and Phase II efficacy assessments based on contact may be overlooking important questions on the coverage and dose needed to achieve long-term efficacy such as: would reducing the total dose be possible if a larger surface was treated? Phase II early morning collections do offer an insight on the question, as not all wild mosquitoes entering the hut are in direct contact with treated surfaces, but the exact distances from treated surfaces would not be known, numbers would vary between huts and whether the insecticide is on the walls or bed nets might make a difference. Likewise, current Phase I and Phase II assessments may not fully explore the potential of high vapour pressure insecticides by evaluating efficacy chiefly upon contact as opposed to both, contact and distance.

The tested insecticide product was effective in killing mosquitoes that had not come closer than $1 \mathrm{~m}$ to the treated surface after an overnight exposition. It could be envisaged that the same could happen in a natural setting to mosquitoes resting on non-treated surfaces before and/ or after biting, although it is not known the minimum amount of exposure time needed to achieve this. A study performed by Gimnig et al. [17] determined how the abundance of malaria vectors changed as a function of distance from houses with ITNs. The study used a geographic information system (GIS) to test the hypothesis that a community effect reduces the overall vector population, and that persons lacking ITNs who live near the compounds of those using ITNs are afforded some protection from vector mosquitoes. Another study performed by Hawley et al. [30] showed ITNs had a protective effect on child mortality, moderate anaemia, high-density parasitaemia, and haemoglobin levels in compounds lacking ITNs but located within $300 \mathrm{~m}$ of compounds with ITNs. A mass community effect against malaria transmission has also been observed in areas where the only malaria vector is largely exophagic and zoophilic [31].

In the case of dengue, a spatial analysis performed by Lenhart et al. [32] indicated that the effect of the presence of ITNs had spread to control houses located $50-100 \mathrm{~m}$ away from bed net houses by five months post-intervention, although control houses located more than $100 \mathrm{~m}$ from bed net houses experienced no significant change in entomological indices. Findings were particularly surprising since there were no previous indications that ITNs could be useful in reducing dengue transmission. Although the nature of that effect was not characterized, the study suggested that it was of both repellent and lethal nature and not by the barrier provided by the ITN itself [32].

Findings increasingly suggest the mass community effect depends on high rates of coverage $[33,34]$ as well as the distance from treated clusters [35]. Despite evidence pointing at the mass community effect of vector control strategies, little is known on the exact mechanism. Contact alone may not be the sole factor. Recent findings emphasize the need to study the spatial repellency of insecticides in addition to the contact irritancy tests classically done [36]. Furthermore, spatial repellency may represent an effective tool in the fight against vectorborne disease transmission [37]. It is proposed that the spatial mortality of insecticide products is evaluated in addition to the contact mortality tests currently done in order to better rationalize vector control efforts. Future endeavours will be directed towards the testing of the 
lethal efficacy at a distance of Inesfly $5 \mathrm{~A} \mathrm{IGR}^{\mathrm{m}}$ against OPresistant An. gambiae in the laboratory and the field.

\section{Conclusions}

Spatial mortality assessments provide additional information overlooked by the contact efficacy tests recommended at present. It is therefore proposed that tests to evaluate the spatial mortality effect of insecticides are added to the battery of Phase I and Phase II WHOPES tests in the laboratory and the field. A tool to evaluate an insecticide product's lethal effect at a distance in the laboratory may be distance boxes although further studies are needed before this method is standardised. In the field, exposing mosquitoes at a fixed distance from treated surfaces may provide valuable information with little added effort. In order to better rationalize integrated vector control strategies, it may be important to assess the full profile of an insecticide by doing both contact and spatial lethal efficacy tests.

\section{Competing interests}

The authors declare that they have no competing interests.

\section{Authors' contributions}

SMC had the idea of performing distance tests as part of the protocol to test for insecticide efficacy. SMC, PC and BM conceived the protocol. PC, SMC and $\mathrm{BM}$ contributed to the design of the study. FC and JMH contributed to the implementation of the study. JC and BM conducted evaluations. MA was the director of the Centre de Recherche Entomologique de Cotonou (CREC). The manuscript has been written by BM and has been revised by SMC and JC. All authors read and approved the final manuscript.

\section{Acknowledgements}

This study was funded by Project No. 11-CAP2-1558 of the Agencia Española de Cooperación Internacional para el Desarrollo (AECID), Ministry of Foreign Affaires, Madrid, Spain, by project ISCIII-RETIC RD12/0018/0013, Red de Investigación de Centros de Enfermedades Tropicales - RICET, of the Program of "Redes Temáticas de Investigación Cooperativa" RETICS/FEDER, Fondo de Investigación Sanitaria (FIS), Ministry of Health and Consumption, Madrid, Spain; and by project PROMETEO/2012/042 of the programme of Ayudas para Grupos de Investigación de Excelencia, Generalitat Valenciana, Valencia, Spain. We are grateful to volunteers at the Ladji Experimental Station for their good work. We thank Lazare Hounkarin for his dedication and technical support at the Ladji Station and CREC. We thank the University of Valencia in Spain, the Institut de Recherche pour le Développement (IRD) in France, and the Centre de Recherche Entomologique de Cotonou (CREC) in Benin for their support.

\section{Author details}

'Departamento de Parasitologia, Facultad de Farmacia, Universidad de Valencia, Av Vicent Andrés Estellés s/n, Burjassot 46100, Valencia, Spain. ${ }^{2}$ Centre de Recherches Entomologique de Cotonou, 06 BP 2604, Cotonou, Benin. ${ }^{3}$ Institut de Recherche pour le Développement (IRD), Laboratoire de Lutte contre les Insectes Nuisibles, BP 64501, Montpellier Cedex 534394 , France. ${ }^{4}$ Immeuble Le Majoral, Avenue de la Tramontane, Portiragnes Plage 34420, France.

Received: 11 June 2013 Accepted: 22 September 2013

Published: 18 October 2013

\section{References}

1. McGraw EA, O'Neill SL: Beyond insecticides: new thinking on an ancient problem. Nat Rev Microbiol 2013, 11:181-193.

2. Lengeler C, Sharp B: Indoor residual spraying and insecticide-treated nets, Reducing malaria's burden: evidence of effectiveness for decision makers. Washington D.C: Global Health Council; 2003:17-24.

3. Lengeler $\mathrm{C}$ : Insecticide-treated bed nets and curtains for preventing malaria. Cochrane Database of Syst Rev 2008. http://www.cochrane.org.
4. WHO: Guidelines for laboratory and field testing of long-lasting insecticidal mosquito nets: document WHO/HTM/NTD/WHOPES/2013.1. Geneva: World Health Organization; 2013.

5. WHO: Guidelines for testing mosquito adulticides for indoor residual spraying and treatment of mosquito nets: document WHO/CDS/NTD/WHOPES/GCDPP/ 2006.3. Geneva: World Health Organization; 2006.

6. WHO: Test procedures for insecticide resistance monitoring in malaria vectors, bio-efficacy and persistence of insecticides on treated surfaces: report of the WHO informal consultation: document WHO/CDS/CPC/MAL/1998.12 pp1-43. Geneva: World Health Organization; 1998.

7. WHO: Report of the WHO informal consultation on the evaluation and testing of insecticides: document WHO/CTD/WHOPES/IC/1996.1. Geneva: World Health Organization; 1996.

8. Chandre F, Darriet F, Duchon S, Finot L, Manguin S, Carnevale P, Guillet P: Modifications of pyrethroid effects associated with $K d r$ mutation in Anopheles gambiae. Med Vet Entomol 2000, 14:81-88.

9. World Health Organization: Guidelines for testing mosquito adulticides for indoor residual spraying and treatment of mosquito nets: WHO/CDS/NTD/ WHOPES/GCDPP/2006.3. Geneva: World Health Organization; 2006:1-60.

10. Darriet F, Guillet P, N'Guessan R, Doannio JMC, Koffi AA, Konan L, Carnevale P: [Impact of resistance of Anopheles gambiae s.s. to permethrin and deltamethrin on the efficacy of impregnated mosquito nets](in French). Med Trop (Mars) 1998, 58:349-354.

11. Darriet F, N'Guessan R, Hougard JM, Traoré-Lamizana M, Carnevale P: Un outil expérimental indispensable à l'évaluation des insecticides: les cases-pièges. Bull Soc Path Exot 2002, 95:299-303.

12. Beach RF, Ruebush TK, Sexton JD, Bright PL, Hightower AW, Breman JG, Mount $\mathrm{DL}$, Oloo AJ: Effectiveness of permethrin impregnated bednets and curtains for malaria control in a holoendemic area of western Kenya. Am J Trop Med Hyg 1993, 49:290-300.

13. Lines J, Myamba J, Curtis CF: Experimental hut trials of permethrinimpregnated mosquito nets and eave curtains against malaria vectors in Tanzania. Med Vet Entomol 1987, 1:37-51.

14. Magesa SM, Wilkes TJ, Mnzava AEP, Njunwa KJ, Myamba J, Kivuyo MVP, Hill $\mathrm{N}$, Lines JD, Curtis CF: Trial of pyrethroid impregnated bednets in an area of Tanzania holoendemic for malaria: part 2: effects on the malaria vector population. Acta Trop 1991, 49:97-108.

15. Maxwell C, Myamba J, Njunwa KJ, Greenwood BM, Curtis CF: Comparison of bednets impregnated with different pyrethroids for their impact on mosquitoes and on re-infection with malaria after clearance of preexisting infections with chloroproguanil- dapsone. Trans $R$ Soc Trop Med Hyg 1999, 93:4-11.

16. Maxwell CA, Msuya E, Sudi M, Njunwa KJ, Carneiro IA, Curtis CF: Effect of community-wide use of insecticide-treated nets for 3-4 years on malarial morbidity in Tanzania. Trop Med Int Health 2002, 7:1003-1008.

17. Gimnig JE, Kolczak MS, Hightower AW, Vulule JM, Schoute E, Kamau L, Phillips-Howard PA, Kuile FO, Nahlen BL, Hawley WA: Effect of permethrintreated bed nets on the spatial distribution of malaria vectors in western Kenya. Am J Trop Med Hyg 2003, 68(Suppl 4):115-120.

18. Smith T, Hii JLK, Genton B, Muller I, Booth M, Gibson N, Narara A, Alpers A: Associations of peak shifts in age prevalence for human malarias with bednet coverage. Trans R Soc Trop Med Hyg 2001, 95:1-6.

19. Howard SC, Omumbo J, Nevill C, Some ES, Donnelly CA, Snow RW: Evidence for a mass community effect of insecticide-treated bednets on the incidence of malaria on the Kenyan coast. Trans $R$ Soc Trop Med Hyg 2000, 94:357-360.

20. Binka FN, Indome F, Smith T: Impact of spatial distribution of permethrinimpregnated bed nets on child mortality in rural northern Ghana. Am J Trop Med Hyg 1998, 59:80-85.

21. Curtis CF, Mnzava AEP: Comparison of house spraying and insecticidetreated nets for malaria control. Bull World Health Organ 2000, 78:1389-1400.

22. Spanish Ministry of Health and Consumer Affairs: Report on the study of the toxicity and irritability of Inesfly 5A. Madrid: Health Institute Carlos III; 1996.

23. International center of training and medical investigations (CIDEIM): Toxicity studies on Inesfly 5A IGR. Cali (Colombia): CIDEIM; 2003. http://www.cideim.org.co.

24. National center of tropical diseases (CENETROP), santa cruz de la sierra (Bolivia). http://www.cenetrop.org.bo.

25. Amelotti I, Catalá SS, Gorla DE: Experimental evaluation of insecticidal paints against Triatoma infestans (Hemiptera: Reduviidae), under natural climatic conditions. Parasit Vectors 2009, 2:30. 
26. Dias JC, Jemmio A: About an insecticidal paint for controlling Triatoma infestans, in Bolivia. Rev Soc Bras Med Trop 2008, 41:79-81.

27. Mosqueira B, Duchon S, Chandre F, Hougard JM, Carnevale P, Mas-Coma S: Efficacy of an insecticide paint against insecticide-susceptible and resistant mosquitoes - part 1: laboratory evaluation. Malar J 2010, 9:340.

28. Mosqueira B, Chabi J, Chandre F, Akogbeto M, Hougard JM, Carnevale P, Mas-Coma S: Efficacy of an insecticide paint against malaria vectors and nuisance in West Africa: part II: field evaluations. Malar J 2010, 9:341.

29. Darriet F, N'Guessan R, Carnevale P: Evaluation in test huts of the protective effects of untreated mosquito nets against Anopheles gambiae s.s. bites. Cahiers Santé 2000, 10:413-417.

30. Hawley WA, Phillips-Howard PA, Feiko O, Kuile T, Terlouw DJ, Vulule JM, Ombok M, Nahlen BL, Gimnig JE, Kariuki SK, Kolczac MS, Hightower AW: Community-wide effects of permethrin-treated bed nets on child mortality and malaria morbidity in Western Kenya. Am J Trop Med Hyg 2003, 68(suppl 4):121-127.

31. Charlwood JD, Alcantara J, Pinto J, Sousa CA, Rompao H, Gil V, Rosario VE: Do bednets reduce malaria transmission by exophagic mosquitoes? Trans R Soc Trop Med Hyg 2005, 99:901-904.

32. Lenhart A, Orelus N, Maskill R, Alexander N, Streit T, McCall PJ: Insecticidetreated bednets to control dengue vectors: preliminary evidence from a controlled trial in Haiti. Trop Med Int Health 2008, 13:56-67.

33. Teklehaimanot A, Sachs JD, Curtis C: Malaria control needs mass distribution of insecticidal bed nets. Lancet 2007, 369:2143-2146.

34. Vanlerberghe $V$, Trongtokit $Y$, Jirarojwatana $S$, Jirarojwatana $R$, Lenhart $A$, Apiwathnasorn C, McCall PJ, Van der Stuyft P: Coverage-dependent effect of insecticide-treated curtains for dengue control in Thailand. Am J Trop Med Hyg 2013, 89:93-98.

35. Kroeger A, Lenhart A, Ochoa M, Villegas E, Levy M, Alexandrer N, McCall PJ: Effective control of dengue vectors with curtains and water container covers treated with insecticide in Mexico and Venezuela: cluster randomized trials. BMJ 2006, 332:1247-1252.

36. Grieco JP, Achee NL, Chareonviriyaphap T, Suwonkerd W, Chauhan K, Sardelis MR, Roberts DR: A new classification system for the actions of IRS chemicals traditionally used for malaria control. PLOS ONE 2007, 2:e716. doi:10.1371/journal.pone.0000716

37. Achee NL, Bangs MJ, Farlow R, Killeen GF, Lindsay S, Logan JG, Moore SJ, Rowland M, Sweeney K, Torr SJ, Zwiebel L, Grieco JP: Spatial repellents: from discovery and development to evidence-based validation. Malar $J$ 2012, 11:164.

doi:10.1186/1475-2875-12-366

Cite this article as: Mosqueira et al:: Proposed use of spatial mortality assessments as part of the pesticide evaluation scheme for vector control. Malaria Journal 2013 12:366.

\section{Submit your next manuscript to BioMed Central and take full advantage of:}

- Convenient online submission

- Thorough peer review

- No space constraints or color figure charges

- Immediate publication on acceptance

- Inclusion in PubMed, CAS, Scopus and Google Scholar

- Research which is freely available for redistribution 